\title{
Sliding mode control of photoelectric tracking platform based on the inverse system method
}

\author{
Zong Chen Yao ${ }^{1, a}$, He Zhang ${ }^{1, b}$ \\ ${ }^{1}$ School of Mechanical Engineering, Nanjing University of Science and Technology, Nanjing 210094, China
}

\begin{abstract}
In order to improve the photoelectric tracking platform tracking performance, an integral sliding mode control strategy based on inverse system decoupling method is proposed. The electromechanical dynamic model is established based on multi-body system theory and Newton-Euler method. The coupled multi-input multi-output (MIMO) nonlinear system is transformed into two pseudo-linear single-input single-output (SISO) subsystems based on the inverse system method. An integral sliding mode control scheme is designed for the decoupled pseudo-linear system. In order to eliminate system chattering phenomenon caused by traditional sign function in sliding-mode controller, the sign function is replaced by the Sigmoid function. Simulation results show that the proposed decoupling method and the control strategy can restrain the influences of internal coupling and disturbance effectively, and has better robustness and higher tracking accuracy.
\end{abstract}

\section{Introduction}

Photoelectric tracking platform is a two-axis gimbal system equipped with photoelectric detection equipment, can capturing and real-time tracking of moving target in the air or on the ground, has been widely used in military and civil areas.

During tracking, small angle errors can cause big deviation of target position. However, Due to the presence of strong coupling and nonlinear factors in the platform, the tracking performance and accuracy will be affected. Obviously, the problem of coupling and nonlinear need to be resolved urgently and the key lies in the decoupling method. As a nonlinear coupling system, previous literature[1] did not pay enough attention to the coupling factors of the system, it usually treated as disturbances and use the disturbance observer to compensate.

In recent years, various types of decoupling method have been proposed. One is the Intelligent decoupling method which does not depend on the precise mathematical model, such as neural network decoupling[2], sliding mode decoupling[3] and fuzzy decoupling[4] etc. When a system is unable to modeling, theoretically, is more suitable for the use of intelligent decoupling method. However, all the intelligent decoupling method requires a lot of computer resources on practical application, for example, neural network decoupling requires a large amount of measurement data and repeated experiments to optimize the parameters. Another kind of decoupling method is linearization and decoupling (L\&D) method, including inverse system method and differential geometry method. Compared with the differential geometry method, the inverse system method does not need complex nonlinear coordinate transformation, also does not need to transform the nonlinear control problems into the "geometric domain"[5]. Based on these advantages, the inverse system method is more available in practical application.

The tracking accuracy and robust performances can adversely affected by the exist of uncertainties and model errors[6] as well. Therefore, the introduction of a control strategy is required to overcome the shortcomings. Sliding mode control with advantages of whole robustness on external disturbance and uncertainty which satisfy the matching conditions, has been widely used in air vehicle, robot control field and many other complex system control with disturbance and uncertainty $[7,8]$. The introduction of integral can decrease the steady-state error caused by traditional sliding mode, and improve the transient performance of the system[9].

In this paper, a sliding mode control strategy based on inverse system decoupling method for the photoelectric tracking platform with nonlinear coupling, parameter uncertainties and unknown disturbances is proposed. Kinematic relations of the photoelectric tracking platform is analyzed first, and the electromechanical system model of the photoelectric tracking platform is established by using the traditional Newton - Euler method. Based on the inverse system method, the coupling and nonlinearity in system dynamic are resolved, such that the dynamics of two gimbals can be regulated independently. Based on the decoupled pseudo linear system, the integral sliding mode control is applied to reject the influence of uncertainties and disturbances. Moreover, the symbolic function is replaced by the proposed Sigmoid function to

\footnotetext{
a yaozongchen@gmail.com ${ }^{\mathrm{b}}$ hezhangz@njust.edu.cn
} 
restrained the chattering phenomenon of sliding-mode controller. At last, the stability of the proposed control strategy is verified by the Lyapunov criterion. Simulation results demonstrate the effectiveness and reliability of the proposed control strategy.

\section{Modeling of the photoelectric tracking platform}

\subsection{Kinematic relations}

The structure of the photoelectric tracking platform is a two-axis gimbal system. Figure 1 shows the coordinate systems of the photoelectric tracking platform. Three coordinate frames have been introduced as:

1) The base coordinate system $\left\{\mathrm{C}_{1}\right\}$ is fixed on the base or ground.

2) The azimuth gimbal coordinate system $\left\{\mathrm{C}_{2}\right\}$ can rotate around the axis of $\mathrm{OZ}_{2}\left(\mathrm{OZ}_{1}\right)$ and the relative azimuth angle is defined as $\xi$.

3) The elevation gimbal coordinate system $\left\{\mathrm{C}_{3}\right\}$ can rotate around the axis of $\mathrm{OY}_{3}\left(\mathrm{OY}_{2}\right)$ and the relative elevation angle is defined as $\eta$.

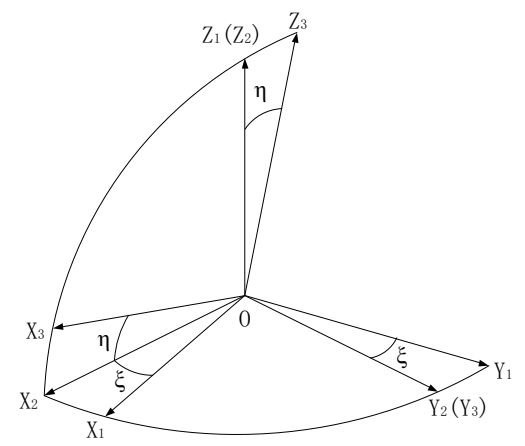

Figure 1. Coordinate systems of the photoelectric tracking platform

By using Euler matrix, transformation matrix from $\left\{\mathrm{C}_{3}\right\}$ to $\left\{\mathrm{C}_{2}\right\}$ become

$$
\mathbf{C}_{e a}=\left[\begin{array}{ccc}
\cos \eta & 0 & \sin \eta \\
0 & 1 & 0 \\
-\sin \eta & 0 & \cos \eta
\end{array}\right]
$$

The relative angular rate of azimuth gimbal and elevation gimbal can be described as:

$$
\begin{aligned}
& \boldsymbol{\omega}_{a a}=\left[\begin{array}{lll}
0 & 0 & \dot{\xi}
\end{array}\right]^{T} \\
& \boldsymbol{\omega}_{e e}=\left[\begin{array}{lll}
0 & \dot{\eta} & 0
\end{array}\right]^{T}
\end{aligned}
$$

By using transformation matrix $\mathbf{C}_{e a}$, absolute angular rate of azimuth gimbal and elevation gimbal becomes:

$$
\begin{gathered}
\boldsymbol{\omega}_{a}=\left[\begin{array}{l}
\omega_{a x} \\
\omega_{a y} \\
\omega_{a z}
\end{array}\right]=\boldsymbol{\omega}_{a a}=\left[\begin{array}{l}
0 \\
0 \\
\dot{\xi}
\end{array}\right] \\
\boldsymbol{\omega}_{e}=\left[\begin{array}{c}
\omega_{e x} \\
\omega_{e y} \\
\omega_{e z}
\end{array}\right]=\mathbf{C}_{e a} \boldsymbol{\omega}_{a}+\boldsymbol{\omega}_{e e}=\left[\begin{array}{c}
-\dot{\xi} \sin \eta \\
\dot{\eta} \\
-\dot{\xi} \cos \eta
\end{array}\right]
\end{gathered}
$$

Where subscript e stands for the elevation gimbal and subscript a stands for the azimuth gimbal; subscripts $\mathrm{x}, \mathrm{y}$ and $\mathrm{z}$ stand for the components in direction of axis $\mathrm{x}$, axis $y$ and axis $z$ of the variables respectively.

\subsection{Dynamical model}

Assuming that each gimbal can be regarded as a rigid body, based on the traditional Newton-Euler equations[10], the dynamic model of photoelectric tracking platform can obtained:

$$
\mathbf{M}=\mathbf{J} \dot{\boldsymbol{\omega}}+\boldsymbol{\omega} \times(\mathbf{J} \boldsymbol{\omega})
$$

Where M stands for the external torque applied to each gimbal; $\mathbf{J}$ stands for the inertia moments; $\boldsymbol{\omega}$ stands for absolute angular rate ; $\dot{\boldsymbol{\omega}}$ stands for absolute angular acceleration.

The equation also can be written as:

$$
\mathbf{M}=\left[\begin{array}{l}
M_{x} \\
M_{y} \\
M_{z}
\end{array}\right]=\left[\begin{array}{c}
J_{x} \dot{\omega}_{x} \\
J_{y} \dot{\omega}_{y} \\
J_{z} \dot{\omega}_{z}
\end{array}\right]+\left[\begin{array}{l}
\left(J_{z}-J_{y}\right) \omega_{y} \omega_{z} \\
\left(J_{x}-J_{z}\right) \omega_{x} \omega_{z} \\
\left(J_{y}-J_{x}\right) \omega_{y} \omega_{x}
\end{array}\right]
$$

Because each gimbal respect to their coordinate axes is axisymmetric, so the product of inertia in inertia moments matrix of each gimbal is zero ${ }^{[11]}$, then the inertia moments $\mathbf{J}$ becomes:

$$
\mathbf{J}=\operatorname{diag}\left[\begin{array}{lll}
J_{x} & J_{y} & J_{z}
\end{array}\right]
$$

The inertia moments respect to the rotation axis of elevation gimbal and azimuth gimbal are given by

$$
\begin{gathered}
J_{O e y}=J_{e y} \\
J_{O a z}=J_{a z}+J_{e x} \sin ^{2} \eta+J_{e z} \cos ^{2} \eta
\end{gathered}
$$

\subsubsection{Elevation gimbal}

The external torque of elevation gimbal can be expressed as

$$
\mathbf{M}_{e}=\left[\begin{array}{c}
M_{e x} \\
M_{e y} \\
M_{e z}
\end{array}\right]=\left[\begin{array}{c}
M_{e x} \\
T_{e}-M_{d e} \\
M_{e z}
\end{array}\right]
$$

Where $M_{e}$ stands for coupling torque between the azimuth gimbal and the elevation gimbal in directions of each axis; $T_{e}$ is the motor torque of the elevation gimbal; $M_{d e}$ represent uncertainties and disturbance including friction torque, unmodeled dynamics, wire torque etc.

By combination of (7) and (11), the dynamic model of the elevation gimbal can obtained

$$
\left[\begin{array}{c}
M_{e x} \\
T_{e}-M_{d e} \\
M_{e z}
\end{array}\right]=\left[\begin{array}{c}
J_{e x} \dot{\omega}_{e x} \\
J_{e y} \dot{\omega}_{e y} \\
J_{e z} \dot{\omega}_{e z}
\end{array}\right]+\left[\begin{array}{l}
\left(J_{e z}-J_{e y}\right) \omega_{e y} \omega_{e z} \\
\left(J_{e x}-J_{e z}\right) \omega_{e x} \omega_{e z} \\
\left(J_{e y}-J_{e x}\right) \omega_{e y} \omega_{e x}
\end{array}\right]
$$

The elevation gimbal only has rotational freedom in direction of axis y, therefore, take its component of axis y, and the dynamic model of elevation gimbal become

$$
T_{e}-M_{d e}=J_{e y} \dot{\omega}_{e y}+\left(J_{e x}-J_{e z}\right) \omega_{e x} \omega_{e z}
$$

\subsubsection{Azimuth gimbal}

Due to the reaction moment from the elevation gimbal, the dynamic model of the azimuth gimbal become

$$
\mathbf{M}_{a}=\mathbf{J}_{a} \dot{\boldsymbol{\omega}}_{a}+\boldsymbol{\omega}_{a} \times\left(\mathbf{J}_{a} \boldsymbol{\omega}_{a}\right)+\mathbf{M}_{a e}
$$

Where $\mathbf{M}_{a e}$ stands for the reaction moment from the elevation gimbal, and it can be obtained by using the transformation matrix $\mathbf{C}_{e a}$ :

$$
\mathbf{M}_{a e}=\mathbf{C}_{e a}^{-1} \mathbf{M}_{e}
$$


The external torque of azimuth gimbal can be expressed as

$$
\mathbf{M}_{a}=\left[\begin{array}{c}
M_{a x} \\
M_{a y} \\
M_{a z}
\end{array}\right]=\left[\begin{array}{c}
M_{a x} \\
M_{a z} \\
T_{a}-M_{d a}
\end{array}\right]
$$

By combination of (14) and (16), the dynamic model of the azimuth gimbal can obtained:

$$
\left[\begin{array}{c}
M_{a x} \\
M_{a z} \\
T_{a}-M_{d a}
\end{array}\right]=\left[\begin{array}{c}
J_{a x} \dot{\omega}_{a x} \\
J_{a y} \dot{\omega}_{a y} \\
J_{a z} \dot{\omega}_{a z}
\end{array}\right]+\left[\begin{array}{c}
\left(J_{a z}-J_{a y}\right) \omega_{a y} \omega_{a z} \\
\left(J_{a x}-J_{a z}\right) \omega_{a x} \omega_{a z} \\
\left(J_{a y}-J_{a x}\right) \omega_{a y} \omega_{a x}
\end{array}\right]+\mathbf{M}_{a e}
$$

The azimuth gimbal only has rotational freedom in direction of axis $z$, therefore, take its component of axis $z$, and the dynamic model of azimuth gimbal become

$$
T_{a}-M_{d a}=J_{a z} \dot{\omega}_{a z}+\left(J_{a y}-J_{a x}\right) \omega_{a y} \omega_{a x}+M_{a e z}
$$

\subsubsection{Driving torque of motors}

The pivoting movement of each gimbal were achieved by torque motors, therefore, the dynamic model of torque motors[12] for azimuth gimbal and elevation gimbals can be described as

$$
\left\{\begin{array}{l}
T_{a}=\frac{K_{T a}}{R_{a}}\left(U_{a}-n_{a} K_{a} \dot{\xi}\right) \\
T_{e}=\frac{K_{T e}}{R_{e}}\left(U_{e}-n_{e} K_{e} \dot{\eta}\right)
\end{array}\right.
$$

Where $T$ stand for the electromagnetic torque generated by motor; $K_{T}$ stand for the torque coefficient of the motor; $R$ stand for the armature resistance of the motor; $U$ stand for the input driven voltage of the motor. $n$ stand for the reduction ratio of the reducer; $K$ stand for back EMF constant.

Finally, the electromechanical dynamic model of the photoelectric tracking platform can obtained

$$
\left\{\begin{array}{l}
\frac{K_{T a}}{R_{a}}\left(U_{a}-n_{a} K_{a} \dot{\xi}\right)-M_{d a}=J_{O a z} \ddot{\xi}-2\left(J_{e x}-J_{e z}\right) \dot{\eta} \dot{\xi} \sin \eta \cos \eta \\
\frac{K_{T e}}{R_{e}}\left(U_{e}-n_{e} K_{e} \dot{\eta}\right)-M_{d e}=J_{O e y} \ddot{\eta}-\left(J_{e x}-J_{e z}\right) \dot{\xi}^{2} \sin \eta \cos \eta
\end{array}\right.
$$

\section{Decoupling control}

Before decoupling design, the dynamic model should be transformed to a form of state space representation.

By substituting the inertia moments and motor parameters value from [13], the differential equation of the system can be obtained:

$$
\left\{\begin{array}{l}
\ddot{\xi}=-6.03 \dot{\xi}-0.16 \dot{\eta} \dot{\xi} \sin \eta \cos \eta+0.52 U_{a}+d_{1}(t) \\
\ddot{\eta}=-47.98 \dot{\eta}-0.67 \dot{\xi} \sin \eta \cos \eta+3.26 U+d_{2}(t)
\end{array}\right.
$$

Assuming that :

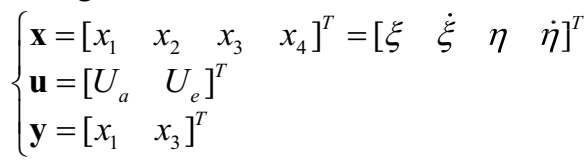

Where $\mathbf{x}$ is a 4-dimensional state vector; $\mathbf{u}$ stand for the control input; $\mathbf{y}$ stand for the control output.

The state space of original system $s_{0}$ can be described as:

$$
s_{0}=\left\{\begin{array}{l}
\dot{\mathbf{x}}=f(\mathbf{x})+b(\mathbf{x}) \mathbf{u}+g(\mathbf{x}) \\
\mathbf{y}=h(\mathbf{x})
\end{array}\right.
$$

Where $b(\mathbf{x})$ stands for the input matrix, $h(\mathbf{x})$ stands for the output matrix:

$$
\begin{gathered}
f(\mathbf{x})=\left[\begin{array}{c}
x_{2} \\
-0.63 x_{2}-0.16 x_{2} x_{4} \sin x_{3} \cos x_{3} \\
x_{4} \\
-47.98 x_{4}-0.67 x_{2} \sin x_{3} \cos x_{3}
\end{array}\right], \\
b(\mathbf{x})=\left[\begin{array}{cc}
0 & 0 \\
0.52 & 0 \\
0 & 0 \\
0 & 3.26
\end{array}\right], h(\mathbf{x})=\left[\begin{array}{ll}
x_{1} & x_{3}
\end{array}\right]^{T}
\end{gathered}
$$

It is observed that the photoelectric tracking platform is a 2-input 2-output system with characteristics of strong coupling and nonlinearity.

\subsection{Decoupling and linearization}

Based on the system model (23), take the derivative of the output $y$ with respect to the time t until the control variables $U_{a}$ and $U_{e}$ is explicitly included, which can get the following equation:

$J(\mathbf{u})=\left[\begin{array}{l}\ddot{y}_{1} \\ \ddot{y}_{2}\end{array}\right]=\left[\begin{array}{l}\dot{x}_{2} \\ \dot{x}_{4}\end{array}\right]=\left[\begin{array}{l}-0.63 x_{2}-0.16 x_{2} x_{4} \sin x_{3} \cos x_{3}+0.52 U_{a} \\ -47.98 x_{4}-0.67 x_{2} \sin x_{3} \cos x_{3}+3.26 U_{e}\end{array}\right]$

The Jacobi matrix of $J(\mathbf{u})$ with respect to the control input vector $\mathbf{u}$ can be calculated as follows:

$$
\mathbf{D}=\frac{\partial J(\mathbf{u})}{\partial \mathbf{u}^{T}}=\left[\begin{array}{cc}
0.52 & 0 \\
0 & 3.26
\end{array}\right]
$$

According to $(26), \operatorname{det}(\mathbf{D}) \neq 0$. Moreover, the relative degrees of the system are $\boldsymbol{\alpha}=\left(\alpha_{a}, \alpha_{e}\right)=(2,2)$, which satisfy $\alpha_{a}+\alpha_{e}=4 \leq n$, where $n$ is the dimensions of the state vector $\mathbf{x}$. Based on the inverse system theory [14], the original system is invertible.

Define the new input variables

$$
\boldsymbol{\varphi}=\left[\begin{array}{l}
\varphi_{1} \\
\varphi_{2}
\end{array}\right]=\left[\begin{array}{c}
\ddot{y}_{1} \\
\ddot{y}_{2}
\end{array}\right]
$$

By substituting (26) into (24) and make $\mathbf{u}=\left[\begin{array}{ll}U_{a} & U_{e}\end{array}\right]^{T}$ as output can get the inverse system $s_{0}^{-1}$

$$
s_{0}^{-1}=\left\{\begin{array}{l}
\dot{\mathbf{x}}=f(\mathbf{x})+b(\mathbf{x}) \mathbf{u}+g(\mathbf{x}) \\
\mathbf{u}=\mathbf{D}^{-1}[\boldsymbol{\varphi}-\bar{f}(\mathbf{x})]
\end{array}\right.
$$

If the inverse system $s_{0}^{-1}$ in series with the original system $s_{0}$ (see Figure 2(a)), two pseudo-linear subsystems can be formed (see Figure 2(b)).

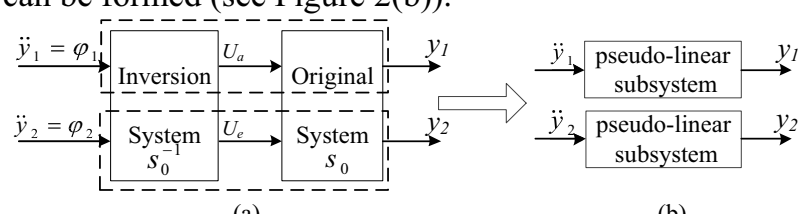

(a)

(b)

Figure 2 decoupling and linearization

\subsection{Controller design}

For the pseudo-linear subsystem, the system uncertainties and external disturbance should be considered, then (27) can be written as:

$$
\boldsymbol{\varphi}=\left[\begin{array}{l}
\varphi_{1}+d_{1} \\
\varphi_{2}+d_{2}
\end{array}\right]=\left[\begin{array}{l}
\ddot{y}_{1} \\
\ddot{y}_{2}
\end{array}\right]
$$


Where $d_{1}$ and $d_{2}$ are the equivalent parameter of system uncertainties and external disturbance, unknown but bounded.

Define the tracking error $\mathbf{e}=\left[\begin{array}{l}e_{a} \\ e_{e}\end{array}\right]=\left[\begin{array}{l}x_{1}-r_{1} \\ x_{3}-r_{3}\end{array}\right]$, in which $r_{1}$ and $r_{3}$ represents the reference trajectory.

Accordingly,

$\dot{\mathbf{e}}=\left[\begin{array}{c}\dot{e}_{a} \\ \dot{e}_{e}\end{array}\right]=\left[\begin{array}{c}\dot{x}_{1}-\dot{r}_{1} \\ \dot{x}_{3}-\dot{r}_{3}\end{array}\right] \ddot{\mathbf{e}}=\left[\begin{array}{c}\ddot{e}_{a} \\ \ddot{e}_{e}\end{array}\right]=\left[\begin{array}{c}\ddot{x}_{1}-\ddot{r}_{1} \\ \ddot{x}_{3}-\ddot{r}_{3}\end{array}\right]=\left[\begin{array}{c}\varphi_{1}-\ddot{r}_{1}+d_{1} \\ \varphi_{2}-\ddot{r}_{3}+d_{2}\end{array}\right]$

Then the sliding mode surface with integral actions[15] is designed as follows

$$
\mathbf{S}=\left[\begin{array}{l}
S_{a} \\
S_{e}
\end{array}\right]=\left[\begin{array}{l}
\left(c_{1}+c_{2}\right) \mathrm{e}_{a}+c_{1} c_{2} \int_{0}^{t} e_{a} d \tau+\dot{e}_{a} \\
\left(c_{3}+c_{4}\right) \mathrm{e}_{a}+c_{3} c_{4} \int_{0}^{t} e_{e} d \tau+\dot{e}_{e}
\end{array}\right]
$$

And the control input $\boldsymbol{\varphi}$ is designed as

$\left[\begin{array}{c}\varphi_{1} \\ \varphi_{2}\end{array}\right]=\left[\begin{array}{l}\ddot{r}_{1}-\left(c_{1}+c_{2}\right) \dot{\mathrm{e}}_{a}+c_{1} c_{2} e_{a}-k_{1} S_{a}-k_{2} \operatorname{sign}\left(S_{a}\right) \\ \ddot{r}_{3}-\left(c_{3}+c_{4}\right) \dot{\mathrm{e}}_{e}+c_{3} c_{4} e_{e}-k_{3} S_{e}-k_{4} \operatorname{sign}\left(S_{e}\right)\end{array}\right]$

Where $c_{1} c_{2} c_{3}$ and $c_{4}$ are positive constant, $k_{1}$ and $k_{3}$ are fixed gain which satisfied with $k_{1}>0$ and $k_{3}>0, k_{2}$ and $k_{4}$ are switching gain which satisfied with $k_{2}>\left|d_{1}\right|, k_{4}>\left|d_{2}\right|$. The control block diagram is shown in Figure 3.

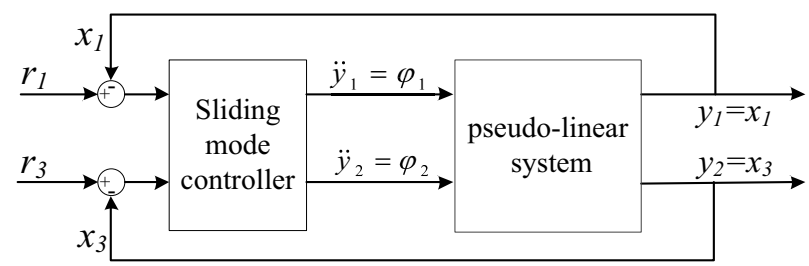

Figure 3. Control block diagram

\subsection{Stability analysis}

For the stability analysis of the system dynamics, select a Lyapunov function as

$$
V=\frac{1}{2} S^{2}
$$

The time derivative of $V$ is

$$
\begin{aligned}
\dot{\mathbf{V}} & =\mathbf{S} \mathbf{T} \dot{\mathbf{S}}=S_{a}\left[\left(c_{1}+c_{2}\right) \dot{e}_{a}+c_{1} c_{2} e_{a}+\ddot{e}_{a}\right]+S_{e}\left[\left(c_{3}+c_{4}\right) \dot{e}_{e}+c_{3} c_{4} e_{e}+\ddot{e}_{e}\right] \\
& =S_{a}\left[\left(c_{1}+c_{2}\right) \dot{e}_{a}+c_{1} c_{2} e_{a}+\varphi_{1}-\ddot{r}_{1}+d_{1}\right] \\
& +S_{e}\left[\left(c_{3}+c_{4}\right) \dot{e}_{e}+c_{3} c_{4} e_{e}+\varphi_{2}-\ddot{r}_{2}+d_{2}\right]
\end{aligned}
$$

Substituting (31) into (32), (32) becomes

$$
\begin{aligned}
\dot{\mathbf{V}} & =\mathbf{S}^{\mathrm{T}} \dot{\mathbf{S}}=S_{a}\left[-k_{1} S_{a}-k_{2} \operatorname{sign}\left(S_{a}\right)+d_{1}\right]+S_{e}\left[-k_{3} S_{e}-k_{4} \operatorname{sign}\left(S_{a}\right)+d_{2}\right] \\
& \leq-k_{1} S_{a}^{2}-\left(k_{2}-d_{1}\right)\left|S_{a}\right|+\left(-k_{3} S_{e}^{2}-\left(k_{4}-d_{2}\right)\left|S_{e}\right|\right) \leq 0
\end{aligned}
$$

From Lyapunov stability theory, it means the designed control system is stable, and the system can reach the sliding mode surface in finite time.

\subsection{Chattering restrain}

We can see from (31) that the control law $\boldsymbol{\varphi}$ contains the symbolic function sign(). Although due to the existence of it can ensure the robustness of sliding mode control, it can also led to the chattering phenomenon of sliding mode control, affecting the convergence precision of the system, even can inspiring the unmodeled dynamics and make the system unstable. In order to weaken the chattering phenomenon and strengthen the robustness of system at the same time, the Sigmoid function[16] is designed to replace the symbolic function as:

$$
\operatorname{Sig}(\mathrm{x})=\frac{2}{1+\exp (-a x)}-1
$$

Where $a$ is positive constant, it determines the convergence rate of the sigmoid function.

\section{Simulation results}

To verify the effectiveness and reliability of the decoupling control strategy proposed in this paper, simulations have been carried out on the photoelectric tracking platform.

The initial position of two gimbals is set as $\mathbf{x}_{0}=\left[\begin{array}{ll}0 & 0\end{array}\right]^{T}$, the tracking signals are given as $r_{1}=\pi-\pi \sin (2 \pi \times 0.3 \mathrm{t}), r_{3}=\pi / 4-\pi / 4 \sin (2 \pi \times 0.2 \mathrm{t})$, and assuming that the disturbance torque of two gimbals are $d_{1}=0.5 \sin (\pi t), d_{2}=0.1 \sin (\pi t)$ respectively.

The simulation results are shown in Figure $4 \sim 9$ :

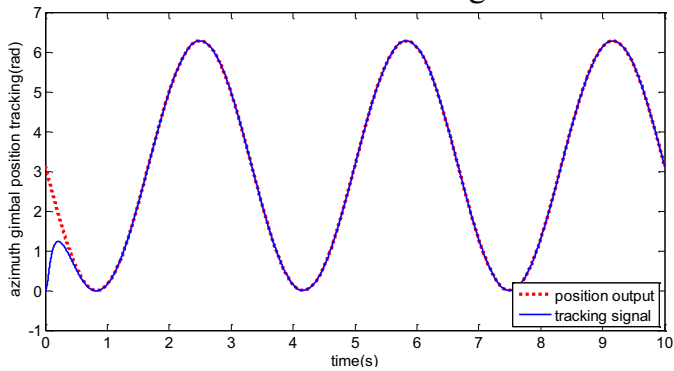

Figure 4. Position tracking of azimuth gimbal

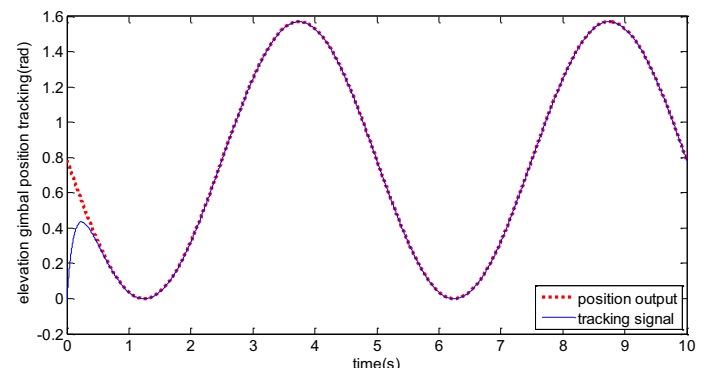

Figure 5. Position tracking of elevation gimbal

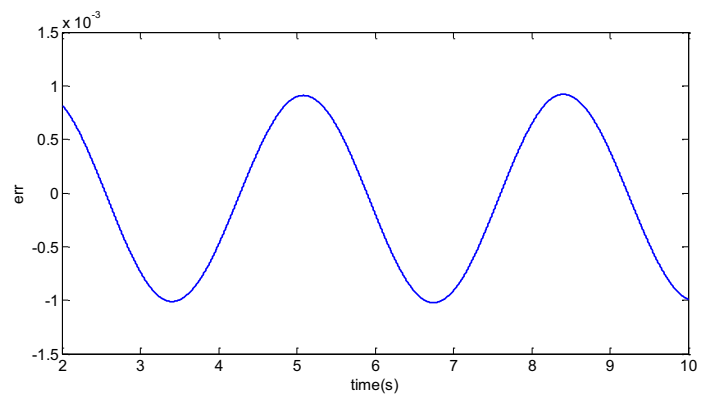

Figure 6. Tracking error of azimuth gimbal(2s $\sim 10 \mathrm{~s})$ 


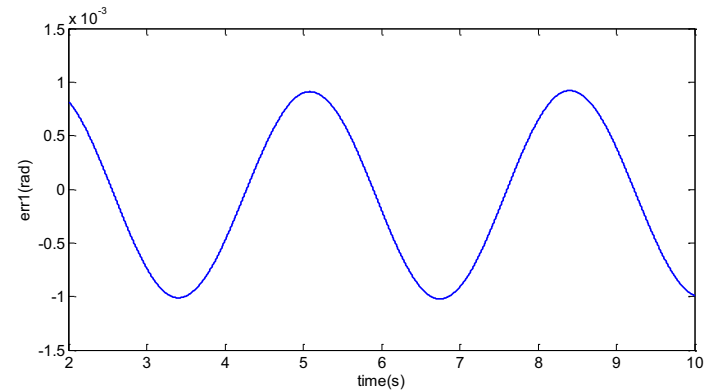

Figure 7. Tracking error of elevation gimbal( $2 \mathrm{~s} \sim 10 \mathrm{~s})$

Figure 4 and 5 are position tracking curves of azimuth gimbal and elevation gimbal respectively, it is observed that the system state can be reaching and perfect tracking to the desired state within 1s Figure 6 and 7 are tracking error curves from $2 \mathrm{~s}$ to $10 \mathrm{~s}$ of azimuth gimbal and elevation gimbal respectively, it is observed that the tracking error of both gimbals are less than $1 \mathrm{mrad}$. It can be concluded that the system has fast convergence speed and dynamic response, can effectively reduce the influence of system uncertainties and disturbance torque, and satisfying the requirements of control precision.

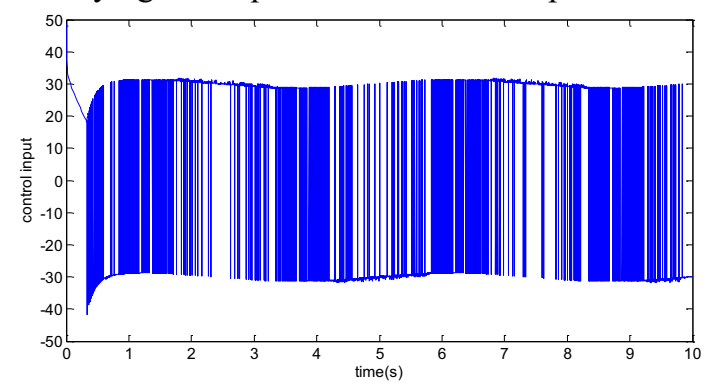

Figure 8 control input signal (using traditional sign $(x)$ )

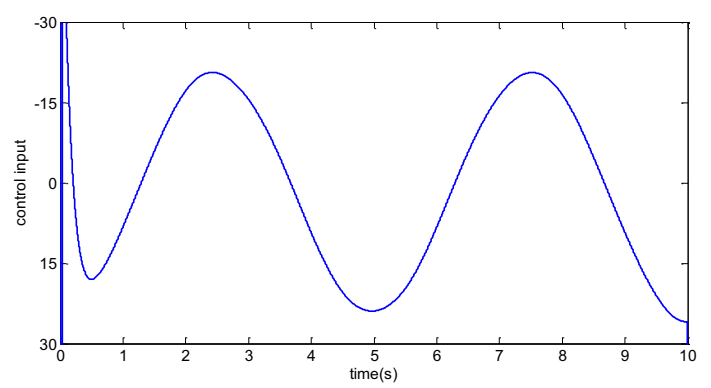

Figure 9. Control input signal (using $\operatorname{Sig}(x)$ )

From Figure 8, it is found that the controller which using the traditional symbolic function $\operatorname{sign}(x)$ has strong chattering phenomenon, and its amplitude can reach more than 30. Figure 8 is control input signal of which the controller using the Sigmoid function $\operatorname{Sig}(x)$. Compared with Figure 9, the chattering phenomenon in Figure 8 has decreased significantly, and its amplitude was controlled at about 2. It can be concluded that the proposed Sigmoid function is able to reduce the chattering phenomenon effectively and ensure the robustness of the system.

\section{Conclusions}

An integral sliding mode control strategy based on inverse system decoupling method is proposed for the photoelectric tracking platform. The kinematic relations is analyzed and the dynamic model is developed based on multi-body system theory and Newton-Euler method. The electromechanical system model are linearized and decoupled into two pseudo linear subsystems and the strong coupling and nonlinearity of the system are resolved. the integral sliding mode controller is designed for the pseudo linear subsystems to reject the influence of system uncertainties and disturbance. By adopting the proposed Sigmoid function, The chattering phenomenon of sliding-mode controller is restrained. The performance of the proposed decoupling control strategy is studied in simulation. It is observed that the proposed decoupling control strategy can effectively restrain the influences of coupling and disturbance, and has satisfactory tracking performance.

\section{References}

1. T. Bhagyashri, S. Kurode, P. Parkhi, 12th International Conference on Control, Automation and Systems 1358 (2012)

2. R.L. McMillen, J.E. Steck, K.Rokhsaz, J. Aircraft, 32, 1088 (1995)

3. S.J. Dodds, A.B. Walker, Int. J. Control, 54, 737 (1991).

4. J. Nie, IEEE T. Fuzzy. Syst. 5, 304 (1997)

5. J. Fang, Y. Ren, IEEE T. Ind. Electron. 58, 4331 (2011)

6. J Fang, Y. Ren IEEE-ASME T. Mech. 17, 1133 (2012)

7. J.K. Liu, F.C. Sun, Control Theory \& Applications, 23, 407 (2007)

8. W. Lu, C. Li, C. Xu, Int. J. Elec. Power, 57, 39 (2014)

9. J.H. Lee, Automatica, 42, 929 (2006)

10. P.J. Kennedy, R.L. Kennedy. IEEE T. Contr. Syst. T. 11, 3(2003)

11. H. Khodadadi, M.R.J. Motlagh, M. Gorji. International Conference on Electrical, Control and Computer Engineering, 223 (2011)

12. B. Kou, S. Cheng, AC servo motor and its control (China Machine Press, Beijing, 2008)

13. K. Li. Research on Dynamics Modeling and Attitude Stability for Floating Unmanned Platform (Nanjing University of Science\&Technology, Nanjing, 2014)

14. X. Dai, D. He, X. Zhang, et al. IEE P-Contr. 148, 125 (2001)

15. V. Utkin, J. Guldner, J. Shi. Sliding mode control in electro-mechanical systems (CRC press, Boca Raton, 2009)

16. H. Kim, J. Son, J. Lee, IEEE T. Ind. Electron. 58, 4069 (2011) 